\section{The efficacy of topical hyaluronic acid in the management of recurrent aphthous ulceration}

Nolan A, Baillie C et al. J Oral Pathol Med 2006; 35: 461-465

Topical hyaluronic acid (HA) significantly reduced ulceration. Recurrent aphthous ulceration (RAU), when unrelated to systemic conditions, may cause significant discomfort. In a UK dental hospital, 120 patients presenting with discomfort from RAU were randomised to HA or placebo gel for its relief. Up to $4 \mathrm{hrs}$ after initial gel application by a clinician, levels of discomfort were recorded, and patients were given a diary to keep for 7 days, and a supply of the appropriate gel.

A significant reduction in soreness was shown in both active and placebo groups, lasting about 30 minutes before gradual return of discomfort, but there was no difference between treatments. A significant reduction in ulcers was noted in both groups after application, and there was a difference in favour of the active gel 4 days after. The authors consider the reductions in discomfort to be a barrier effect irrespective of HA, but conclude that this substance may have a clinical advantage of reducing the number of ulcers.

doi:10.1038/sj.bdj.4814361

\section{PERIODONTOLOGY; CARDIOLOGY}

\section{Bacterial profile and burden of periodontal infection in subjects with a diagnosis of acute coronary syndrome}

Renvert S, Pettersson T et al. J Periodontol 2006; 77: 1110-1119

Survivors with a diagnosis of acute coronary syndrome (ACS) had a higher oral bacterial load than a matched control sample.

There is current interest in the possible association between periodontal and cardiovascular diseases. Consecutive subjects (161) diagnosed with ACS at a Swedish hospital and surviving the attack were matched to controls. Smoking, the major confounder, was assessed in the categories of never, former and current. (When these frequencies are compared, the result is non-significant, but a test of never-smokers against the rest is significant, showing more never-smokers in the control group.)

Periodontal examination largely within 2 months of hospital release showed moderately but significantly greater disease in the ACS group. However, plaque scores were also greater in this group. Subgingival plaque samples from the 4 deepest sites in 146 ACS and 120 control subjects, where probing depths of $6+\mathrm{mm}$ were present, showed significantly greater load in the ACS group. The authors consider that the load of certain organisms identified in ACS patients may be risk factors for the condition.

\section{Comparison of ultrasound, digital and conventional radiography in differentiating periapical lesions}

Gundappa M, Ng et al. Dentomaxillofac Radio/ 2006; 35: 326-333

Ultrasound underestimated the extent of lesions, but was able to distinguish between cysts and granulomata.

Apical surgery is not considered necessary for many endodontic lesions, but lesions which persist despite re-root treatment are often treated this way. One problem is diagnosis of periapical lesions, which depends on biopsy. In this study, 15 patients requiring endodontic surgery for anterior teeth were given conventional (CR) and digital (DR) periapical radiographs and an ultrasound scan of the teeth. Following surgery, tissue was examined histopathologically.

Radiographic lesion size was assessed by 3 observers who had good agreement for CR and weak agreement for DR, but could not distinguish the nature of lesions. Ultrasound images were given a diagnosis agreed by an ultrasonographer and an endodontist. In 7 cases, the ultrasound diagnosis was of a cyst, and in 8 cases, a granuloma. This agreed exactly with the histopathological diagnosis.

\section{doi:10.1038/sj.bdj.4814362}

\section{PERIODONTOLOGY; CARDIOLOGY}

\section{Periodontal disease and coronary heart disease}

Geismar K, Stoltze K et al. J Periodonto/ 2006; 77: 1547-1554

There was an association between the two conditions in subjects $<60$ yrs old.

Periodontal inflammation and cardiovascular diseases have been shown to be associated in some studies, but there are numerous confounding factors. The present case-control study investigated the relationship in a Copenhagen population, comparing 110 individuals with coronary heart disease (CHD) and 140 healthy controls from a heart study.

Univariate analysis showed the CHD group to have significantly higher levels of diabetes, smoking dose and periodontal disease, but lower levels of high and low density lipoprotein cholesterol. Multiple logistic regression identified diabetes and smoking as significantly associated with CHD, and adjusted odds ratios (ORs) for the relationship of varying levels of alveolar bone to CHD suggested a relationship reaching significance with the highest level of bone loss (6.6; CIs 1.69 to 25.6) only for subjects $<60$ yrs old. In the whole sample, and in those $\geq 60$ years old, the fully adjusted ORs were not significant. 